\title{
Models: stretching the skills of cell lines and mice
}

\author{
Vivien Marx \\ Cell lines are better. Mice are better. Beyond disagreement about model systems and even passionate \\ discord at times, new strategies help to explore the middle ground so models might better approximate \\ human biology.
}

\begin{abstract}
Models such as cell lines or mice are used to portray an aspect of human biology. It is still a matter of "active debate" whether cell lines or animal models are better, says Jeffrey Settleman, who directs oncology discovery at Genentech, which is part of Roche. One system may prove more valuable or predictive in terms of clinical results in certain contexts, but, he says, the general consensus has been that there is no consensus. Rather, both are needed, and they are complementary.

Combining animal and cell-line models and data to address a given scientific question is a way for scientists to exploit the unique viewpoint that each model yields. The 'classic' approach-start experiments with cells, then move to animals to extrapolate to human biology - is shifting as new types of culture systems and strategies emerge along with new challenges.
\end{abstract}

\section{When cell lines talk}

In cancer research, cell culture systems might be a "reasonable place to start," says Levi Garraway, a researcher at Dana-Farber Cancer Institute who is also affiliated with the Broad Institute of Harvard and MIT. That starting point might be probing questions such as tumor survival pathways or resistance mechanisms. And when looking at pathways downstream of a particular genetic alteration, scientists can use cell lines with that very genetic alteration, which might be mutations in the epidermal growth factor receptor (EGFR).

A number of EGFR inhibitors are now on the market to treat patients who have tumors containing EGFR mutations. Ten years ago, says Garraway, if scientists had had cancer cell lines expressing proteins

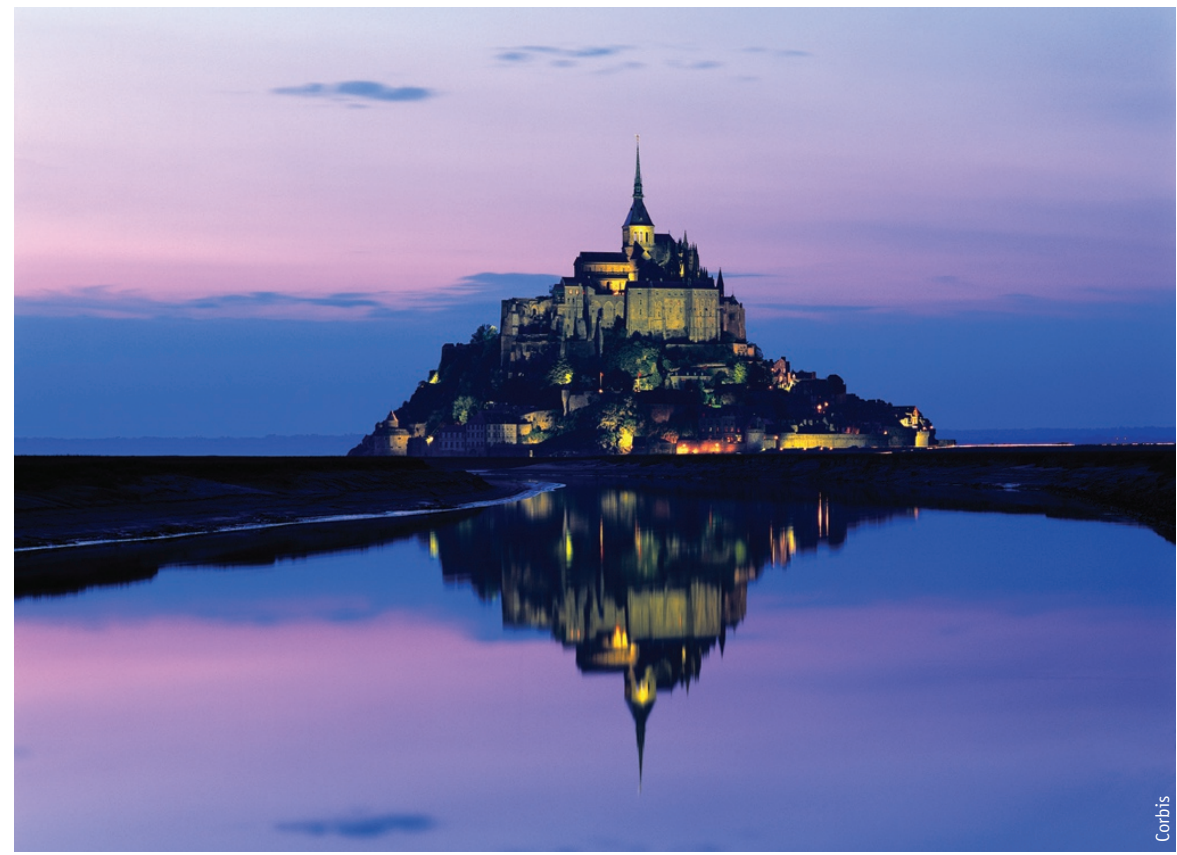

Models can be mirrors. They can also be imperfect representations of what scientists seek to model. Here, Mont Saint-Michel in France, reflected rather perfectly.

with the right genetic characteristics in hand as well as inhibitors geared to target these mutant proteins, a cell-based assay might have "melted away" these tumor cells, he says.

The initial clinical trials could have been targeted to lung cancer patients with EGFR mutations in whom drug response was ultimately found to be successful, says Settleman. But when EGFR inhibitors were in development, cell-line profiling was not routine. The sensitivity of a subset of these patients' tumors was discovered only after much empirical testing ${ }^{1}$.

Today's drug development process benefits from the accelerated knowledge that preclinical cell-line panel testing delivers, says Settleman. Now, unlike ten years ago, many more genomically characterized cells are available, and with them comes the possibility for sensitivity testing on a large scale using 500-1,000 different cell lines from many tumor types and with known mutations.

Many labs have shown that a variety of kinase inhibitors show antitumor activity in patients just as they do in cell lines with the same genomic features. In hindsight, the 'right' cell lines would have allowed researchers to capably predict which of these drugs may be clinically active, says Settleman. Animal models are not an 


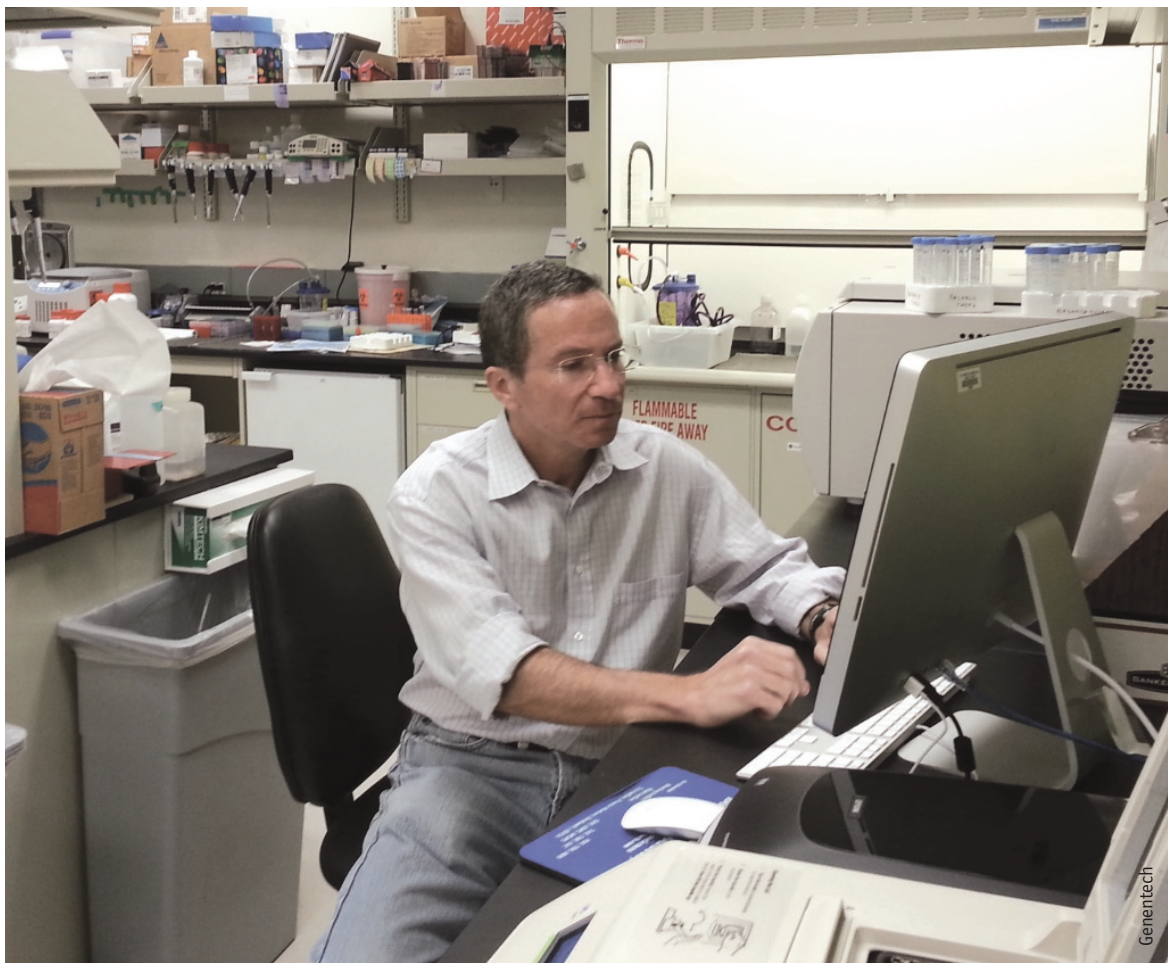

The consensus is that there is no consensus whether cell lines or animal models are more predictive, says Jeffrey Settleman.

"absolute requirement" to predict responsiveness to many drugs. More generally, drug development must still involve animals before clinical trials begin, but these types of cell-line results let researchers focus on mechanisms that underpin the sensitivity of cells to a potential drug.

But even when compounds are on hand that are likely to be effective, killing cells in vitro can be difficult for practical reasons. "Prostate cancer has only a few cell lines that people use," says Garraway. Other cells just do not grow in culture.

Glioblastomas are aggressive brain tumors for which there are limited treatment options. Slightly fewer than half of these tumors have an amplification of the EGFR gene, which appears to make the tumors more aggressive. Not only is it hard to obtain glioblastoma cells from patients, but the cells "don't grow out in standard tissue culture systems," says Garraway.

Researchers need cells with EGFR amplification present in many but not all tumor cells. Having such cells on hand would offer a spectrum of models with the full genetic and molecular diversity seen in this cancer, he says. The diversity would let researchers explore which genetic contexts show sensitivity to which drugs.
Large-scale cell-line characterization projects that document the genetic and drug sensitivity characteristics of cancer cell lines aim to be resources for all researchers: for example, the Cancer Cell Line Encyclopedia project, a collaboration between the Broad Institute, the Novartis Institutes for Biomedical Research and the Genomics Institute of the Novartis Research Foundation ${ }^{2}$.

The Encyclopedia presents sequence data from over 900 cancer cell lines along with genotyping and copy number-variation data for over 450 cell lines. The researchers generated drug sensitivity assays with approved drugs and compounds still under investigation.

Whenever cells are used, authentication is an "essential" practice, says Settleman. Researchers in academic and industry labs must be sure "that they're working with the model they think they're working with" when characterizing molecular and genetic characteristics of cells and testing drugs to validate the genetic factors that could correspond to targets.

\section{Not cells, not organs}

A number of systems are emerging that are neither cells nor animals. For example, Donald Ingber and his team at the Wyss
Institute developed 'organs on chips': threedimensional microfluidic devices combined with cells, which together are physiological and mechanical models of human organs ${ }^{3}$.

In 2009, Hans Clevers and his team at the Hubrecht Institute created cell culture systems called 'organoids' in which cells can grow in a microenvironment ${ }^{4}$. To do so, the scientists leveraged the self-renewing capacity of the small intestine, using sections of mouse small intestine epithelia called crypts, which contain stem cells and immune cells.

Last year, Clevers's team and his longtime colleague Johannes Bos, who runs a center focused on molecular medicine at the University Medical Center Utrecht, received a grant from the foundation Stand Up To Cancer, the American Association for Cancer Research and the Dutch Cancer Society to build a biobank of genetically diverse human tumor organoids for use in preclinical drug testing. Scientists from the Hubrecht Institute, University Medical Center Utrecht, Netherlands Cancer Institute and Wellcome Trust Sanger Institute are part of this project.

Tumor samples from patients are now being grown into organoids with which researchers can explore differences in drug sensitivity or resistance. These types of tests are quick with organoids, says Bos, with either single drugs or drug combinations, and the results can be used, for example, to extrapolate the drug's effect on patient tumors with similar genetic makeup. To study survival pathways, researchers can also apply small hairpin RNAs to silence genes or apply the gene-editing system CRISPR-Cas9, in which clustered, regularly interspaced, short palindromic DNA repeats (CRISPRs) and the nuclease Cas9 are used.

Organoids can fill the gap created by a lack of cell lines-for example, for pancreatic cancer and prostate cancer-to allow these tumors' genetic diversity to be studied, Bos says, because they can be made from various tumors such as prostate, pancreas or colon. Although colon cancer researchers can choose from numerous cell lines that are well suited for studying molecular mechanisms or signal transduction pathways, the genetic diversity of these cell lines is still limited. Organoids are a step "in between" cell lines and mice and are not more difficult to culture than cell lines, says Bos, although they are more expensive owing to the necessary cell culture ingredients. 
Organoids have been kept alive for over two years, says Bos; they are long lived, possibly even immortal. They can be used to study other diseases, too: for example, they might contain the genetic defect specific to cystic fibrosis, says Bos. A researcher can then explore ways to 'rescue' or undo the genetic defect.

Garraway sees organoids as a potential way to "expand your repertoire of models" and to round out systems used to model various genetic or molecular changes common in tumors. As Settleman points out, using organoids means introducing a model that has to be extensively tested and compared. "I think it's a little too early to know how they will stack up against some of these other preclinical models in terms of their predictive ability," he says, referring to cell lines and animal models.

Mouse biologist and geneticist Gary Churchill at The Jackson Laboratory (JAX) calls organoids "a really neat system; I don't think we should ignore it." But, he adds, "I'm not ready to say I'm giving up my mouse."

Heterogeneity is one disease trait that makes modeling challenging. In cancer treatment, clinicians face heterogeneity between tumors in different patients, which can translate to diverse treatment responses, says Settleman. Then there is heterogeneity within a tumor due to its population of tumor cells, nontumor stromal cells and immune cells.

An increased understanding of this heterogeneity has led many to wonder whether an absence of these multiple cell types in cancer cell-line studies truly measures the response to treatment, he says. Some teams counterbalance that absence by coculturing

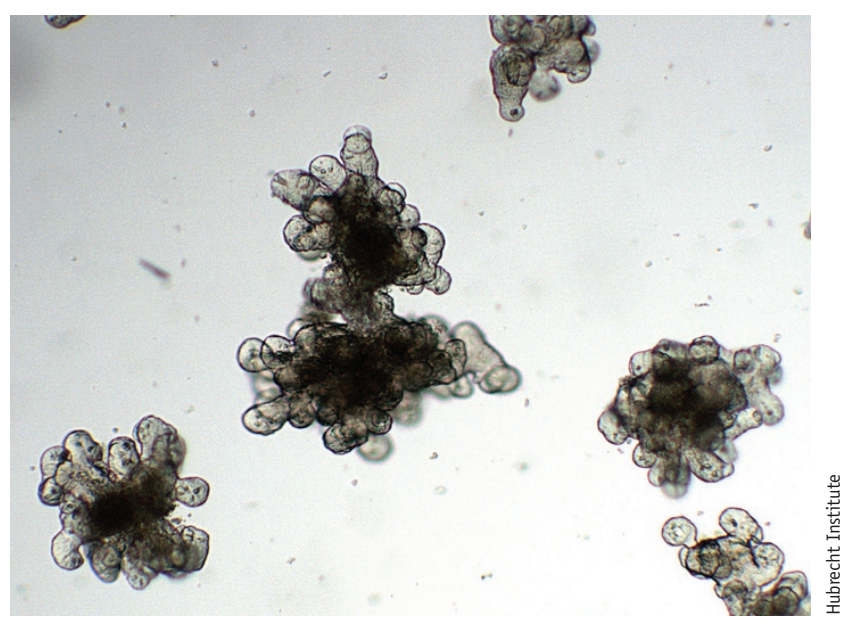

Organoids are a step "in between" cell lines and mice and have been kept alive for over two years, says Johannes Bos. tumor cells and stromal cells in vitro, thereby simulating in vivo heterogeneity without the complications of an animal model.

There is increased interest in profiling epigenetic as well as genomic features of both cell lines and animal models, Settleman says, to help make predictions about which patients are likely to respond to a specific treatment.

\section{Mighty mouse}

In cancer drug development it is common to graft tumor cells onto immunocompromised mice. Some pharmaceutical industry researchers, who declined to be identified, told Nature Methods they are concerned that this immune suppression might be tainting results gained with these animals.

An emerging trend is to use personalized xenograft models in which tumor cells are taken from a patient's tumor and propagated directly in mice. The cells do not see cell culture as an intermediary, says Settleman. With this approach, the heterogeneous tumor is placed in an in vivo setting, but, he says, questions have been raised about the degree of heterogeneity that is maintained over time. It is an open question, he says, whether these xenografts provide a "more faithful model" of human tumors.

Established mouse models can be used to ask new questions. Xenograft experiments usually help determine whether a drug hinders tumor growth. What is not often asked in drug discovery, says Garraway, is whether the particular target is required to maintain an established tumor and whether a drug candidate shrinks the tumor.

Experiments with mice-which may or may not involve genetically engineered animals - to probe aspects such as metastasis or the role of microenvironment and physiology in any disease are "extremely valuable," he says. These experiments deliver completely different information than a cell culture system.

Bos agrees that mice offer a microenvironment in which to study basic physiology or a drug, but "whether this microenviron- ment is sufficiently close to humans to make firm predictions is still a matter of debate." Mice let scientists study physiology, "but it's mouse, it's not human."

The mouseversus-human debate can turn particularly hot. A study last year showed how poorly mice model human biology 5 .

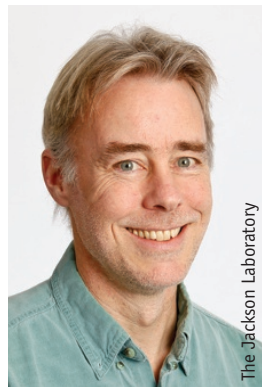

Getting disappointed about a mouse not recapitulating human biology is like saying, "I bought a bike, but it wouldn't fly," says Gary Churchill.
Researchers in a

multicenter project on inflammation compared genome-wide expression responses to endotoxins, burns and trauma in mouse models to the responses of human patients. Some of their motivation heralds from the nearly 150 failed clinical trials for drug candidates that block inflammation in critically ill patients.

The expression in mice and people differed greatly. The authors noted that their results "show that the genomic responses to different acute inflammatory stresses are highly similar in humans, but these responses are not reproduced in the current mouse models" 5 . According to the study authors, contributors to this poor result include the evolutionary distance between mice and humans as well as the inbred mouse model itself.

Churchill views the study with interest because of its lessons and the new questions it raises. The results show that a mouse strain is able to moderate what would be a runaway inflammatory process that can lead to a person's death. "The anomaly is interesting," he says. Perhaps scientists can learn how to nudge the human immune system to behave more like a mouse immune system.

The common lab mouse is inbred. In cancer experiments with these genetically identical mice, all the mice develop tumors at the same time, "like clockwork," says Churchill. These experiments can help cancer researchers parse genomic signals in tumors or diseased tissue.

Researchers need to model whole-body processes and physiology in cancer or in diseases with diverse clinical symptoms, such as diabetes. What begins as blood sugar misregulation can lead to retinopathies, kidney disease and nerve damage. "This doesn't sound like a cell line to me," Churchill says. 


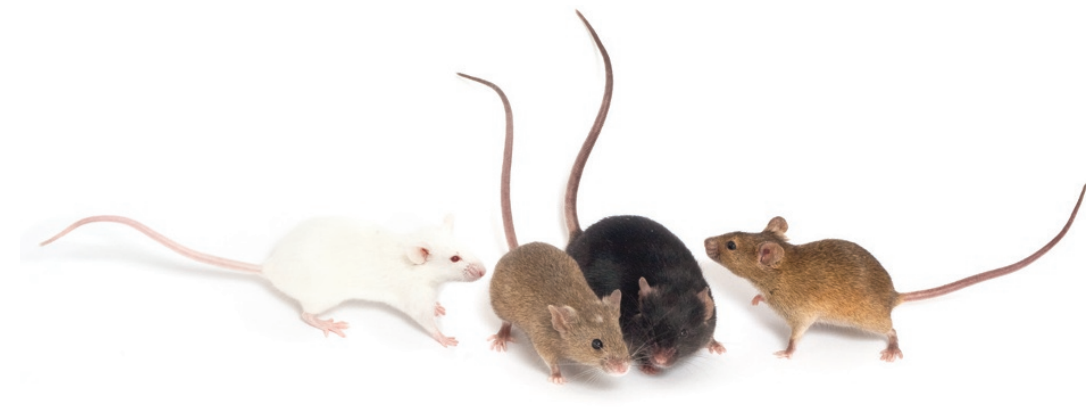

These JAX mice embrace diversity. Here, mice from the Diversity Outbred colony.

Mice, cell lines and organoids are all models that are "imperfect representations of something you are trying to understand," he says. Disappointment about a mouse not recapitulating human biology is almost like saying, "I bought a bike, but it wouldn't fly." Scientists regularly ask Churchill which mouse strain most closely resembles humans biologically, to which he responds: "Which human?" Expecting a mouse to exactly mimic every feature of a human disease is "an unrealistic expectation," he says. What too many experiments with inbred mice fail to do is capture heterogeneity.

In 2001, motivated to model heterogeneity, Churchill set out to "reinvent" the mouse as a tool for biology. He and his team took inbred mice that were part of the Collaborative Cross, a mouse breeding experiment that began at Oak Ridge National Laboratory. From these mice, the JAX team developed the Diversity Outbred (DO) mouse colony, which is now in its 17 th generation. "It gives us basically an endless number of unique mice," he says of the mice that the lab began selling around two years ago and that are mainly used in the academic community.

Churchill and his team genotype their DO mice and use computational modeling to maintain a steady genetic composition and also track genetic drift. They know their mice's 45 million single-nucleotide polymorphisms and 400,000 copy-number variations. To underscore his mice's genetic diversity, Churchill says that, when working with human data, scientists commonly reference around 20 million single-nucleotide polymorphisms.

Using DO mice, the JAX team has explored the side effects of the off-patent chemotherapy drug doxorubicin, which causes cardiac injury in some patients as well as a myelosuppression, which is a reduced white blood cell count. Churchill and his team have been able to recapitulate this myelosuppression in DO mice.

Just as every patient is different, so is each mouse. Not all patients develop myelosuppression, and neither do all DO mice. "The important thing for us is that not all the mice have the same response," he says. This variability lets the team map the genetic factors linked to susceptibility or resistance, which can eventually help explain human biology.

Although scientists can perform gene knockout experiments, it might take many of these experiments to pin down a factor involved in drug toxicity. There are advantages to starting with a biological response in a diverse population and working toward the genetics to understand the mechanism of a given susceptibility, he says.

Inbred mouse strains allow for studies with few animals. With genetically identical animals, a researcher can, for example, study the effect of two different diets. Drug testing with inbred mice, however, is a little like testing a drug on one person. "What you learned about at the end of the day is about the C57 black 6J mouse," Churchill says, referring to a common strainC57BL/6J-used in labs. "As opposed to learning something about mice in general." In experiments with DO mice, diversity is accepted, even embraced, he says.

Recently, he and his team have also begun testing a dozen drug compounds that have failed clinical trials as a way to show drug developers the advantages of working with DO mice. Building diversity into experiments means larger experiments: for example, mice numbers in the low hundreds, Churchill says, to see whether a toxic reaction might occur in a small percentage of animals. Diversity, he says, is a way to capture the range of reactions one might see in a range of people.

Churchill finds it "a little dismaying" when researchers call their own experimental model the only right system. "There's no perfect model," he says. He prefers that researchers use a variety of approaches for their complex problems and explore ways to make each model better. For the mouse, in his view, improvement comes from diversity.

Techniques such as gene editing will prove useful for in vitro and in vivo models, says Garraway, but regardless of technique, a researcher's model choice reverts to the scientific question. Cell lines can be "just fine or even better because of the scalability," he says, allowing for many more experiments than are typically possible in mice. For other questions, mouse models are going to be superior. "Hopefully," says Garraway, "we can move beyond the "my system is better than your system' kind of world."

1. Siegelin, M.D. \& Borczuk, A.C. Lab. Invest. 94, 129-137 (2014).

2. Barretina, J. et al. Nature 483, 603-607 (2012).

3. Huh, D. et al. Science 328, 1662-1668 (2010).

4. Sato, T. et al. Nature 459, 262-265 (2009).

5. Seok, J. et al. Proc. Natl. Acad. Sci. USA 110 3507-3512 (2013).

Vivien Marx is technology editor for Nature and Nature Methods

(v.marx@us.nature.com). 